\title{
Thermal gas rectification using a sawtooth channel
}

\author{
S Solórzano, ${ }^{1}$ N.A.M. Araújo, ${ }^{2,}$, ${ }^{*}$ and H.J. Herrmann ${ }^{1,3}$ \\ ${ }^{1}$ Computational Physics for Engineering Materials, Institut f. Baustoffe (IfB), \\ ETH Zurich, Wolfgang-Pauli-Street 27, 8093 Zurich, Switzerland \\ ${ }^{2}$ Departamento de Física, Faculdade de Ciências, Universidade de Lisboa, \\ P-1749-016 Lisboa, Portugal, and Centro de Física Teórica e Computacional, \\ Universidade de Lisboa, P-1749-016 Lisboa, Portugal \\ ${ }^{3}$ Departamento de Fúsica, Universidade Federal do Ceará, 60451-970 Fortaleza,Ceará, Brazil
}

\begin{abstract}
We study the rectification of a two-dimensional thermal gas in a channel of asymmetric dissipative walls. For an ensemble of smooth Lennard-Jones particles, our numerical simulations reveal a nonmonotonic dependence of the flux on the thermostat temperature, channel asymmetry, and particle density, with three distinct regimes. Theoretical arguments are developed to shed light on the functional dependence of the flux on the model parameters.
\end{abstract}

\section{INTRODUCTION}

Systems where fluctuations are rectified into directed motion are known as Brownian motors or ratchet devices [1, 2]. According to the second law of thermodynamics, these systems ought to be impossible in equilibrium [2, 3]. This is why broken spatial symmetry and non equilibrium conditions are a key feature for their operation [4, 5. Brownian motors are relevant in a number of situations from biological processes to devices for particle segregation [6 9] and transport [10 12]. For instance, asymmetric objects (e.g., wedge shapes) immersed in a granular gas tend to move [13, 14] or rotate [15, 18, in a preferential direction, provided that particle/object collisions are dissipative. Also, granular particles enclosed in a vibrating sawtooth-shaped channel flow along a preferential direction defined by the asymmetry of the channel 19. Similar results are also observed for microscopic particles in an asymmetric channel under the action of a pulsating potential [7]. There are also various examples of active matter systems ranging from bacteria at microscopic scale 20 22, to centimeter scale bots [23, up to pedestrians [24 where rectification can be induced by spatial asymmetries.

Although these systems rectify the motion of the particles or objects immersed within the particle bath, they still require either a pulsating potential or active particles. Further examples, that in some sense relax these requirements, include rectification using differentiated noise sources [25, 26] or asymmetric piston models [2729 that show rectification effects, even when working at a single temperature, provided there is friction and the particle-piston collisions are different on both sides of the piston. So far there are only few examples [30, 31] in which the motion of a single particle in a single dimension is rectified without external driving forces. In the present work, we provide a novel example of collective particle motion rectification in two dimensions. We show that the motion of a gas of Lennard-Jones particles can

\footnotetext{
* nmaraujo@fc.ul.pt
}

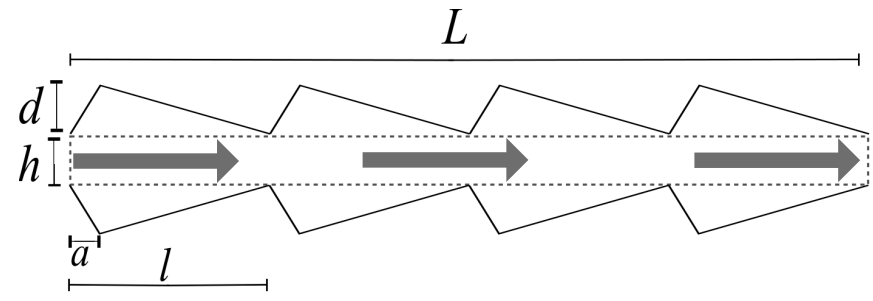

FIG. 1. Schematic representation of the channel of size $L$, with $N=4$ cells. The shape of each cell is characterized by four lengths: the linear length $l$, the aperture size $h$, and the horizontal position $a$ and height $d$ of the peak. The depicted channel is classified as right asymmetric (see text) and the arrows indicate the corresponding direction of particle flow. The dashed lines delimit the region where particles are initially released.

be rectified without external pulsating potentials only by means of dissipation and broken spatial asymmetry. To this end, we consider a two-dimensional gas of particles in a fixed (not moving) asymmetric sawtooth-shaped channel and study how the overall flux depends on the different model parameters. We expect that this idea can find applications in fields such as microfluidic or lab on a chip set ups.

The paper is organized as follows. In Section II the model and methods are introduced. The results are discussed in Section 【II and we draw some conclusions in Section IV.

\section{MODEL AND METHODS}

We consider a two-dimensional sawtooth channel of linear length $L$ consisting of a sequence of $N$ equal cells, as represented in Fig. 1] with periodic boundary conditions along the horizontal axis. The geometry of the channel is characterized by four lengths: the length of each cell $l=L / N$, the aperture size $h$, and the horizontal position $a$ and height $d$ of the edge. To systematically study the dependence on the asymmetry of the channel, we fix $h$ 
and define the adimensional asymmetry coefficient $\alpha$ as,

$$
\alpha=1-2 \frac{a}{l}
$$

where $\alpha \in[-1,1]$. For $\alpha=0$ the channel is symmetric with respect to the vertical axis, while for $\alpha= \pm 1$ the cells look triangular. We classify channels of negative and positive $\alpha$ as left and right asymmetric, respectively.

We consider a gas of particles interacting pairwise, where the force of particle $j$ on particle $i$ is conservative and given by $\mathbf{F}_{i j}=-\nabla_{i} U_{\mathrm{LJ}} . U_{\mathrm{LJ}}$ is the 12-6-LennardJones potential,

$$
U_{\mathrm{LJ}}\left(r_{i j}\right)=4 \epsilon\left[\left(\frac{\sigma}{r_{i j}}\right)^{12}-\left(\frac{\sigma}{r_{i j}}\right)^{6}\right]
$$

where $r_{i j}=\left|\mathbf{r}_{j}-\mathbf{r}_{i}\right|$, and $\mathbf{r}_{i}$ and $\mathbf{r}_{j}$ are the positions of particles $i$ and $j$, respectively. $\epsilon$ corresponds to depth of the potential well which is located at $r_{m}=2^{1 / 6} \sigma$. The force $\mathbf{F}_{\text {iw }}$ of the wall on a particle $i$ is described as the superposition of two contributions: a conservative force, $\mathbf{F}_{\text {iw }}^{c}$, and a dissipative one, $\mathbf{F}_{\text {iw }}^{d}$. The conservative force is described as a Lennard-Jones interaction with the closest point on the wall, with the same $\epsilon$ and $\sigma$ of the particle/particle interaction. The dissipative force is given by,

$$
\mathbf{F}_{\mathrm{iw}}^{d}=-\gamma\left(\dot{\mathbf{r}}_{i} \cdot \hat{\mathbf{n}}_{i w}\right) \hat{\mathbf{n}}_{i w}
$$

where $\hat{\mathbf{n}}_{i w}=\frac{\mathbf{r}_{i}-\mathbf{r}_{w}}{r_{i w}}$ is the unit vector pointing from the closest point on the wall $\mathbf{r}_{w}$ to the particle $i$ and $\gamma \geq 0$ is a friction constant. The particle/wall interaction is conservative for $\gamma=0$ and dissipative otherwise. Interactions with the wall are truncated at a cutoff distance $d_{c}=2.5 \sigma$ and if the particle is within the cutoff distance of multiple points or walls the contributions are superimposed. The particle/wall interaction model was chosen to study the effect of the wall geometry on the particles dynamics. It is assumed that the particles locally bounce off the wall thus the use of a cutoff distance and the nearest point prescription. $d_{c}=2.5 \sigma$ has been found to be a reasonable cut-off Ref. [32]. The analysis of more complex particle/wall interaction models where the walls are directly modelled as a fixed set of particles is left for future work.

We performed canonical molecular dynamics simulations, using the Nosé-Hoover thermostat 32 34. Accordingly, the equation of motion of particle $i$ is,

$$
\begin{aligned}
& \ddot{\mathbf{r}}_{i}=\frac{1}{m_{i}}\left(\sum_{j \neq i} \mathbf{F}_{i j}\left(\mathbf{r}_{i j}\right)+\mathbf{F}_{\mathrm{iw}}\right)+\mathbf{F}_{\mathrm{NH}}, \\
& \mathbf{F}_{\mathrm{NH}}=-\xi \dot{\mathbf{x}},
\end{aligned}
$$

where $\mathbf{r}_{i j}=\mathbf{r}_{j}-\mathbf{r}_{i}, m_{i}$ is the particle mass and $\mathbf{F}_{\mathrm{NH}}$ is the force per unit of mass, resulting from the coupling with the thermostat 34 . $\xi$ is the variable that describes the thermostat and its dynamics is given by

$$
\dot{\xi}=\frac{1}{Q}\left(\sum_{i=1}^{N} \frac{\mathbf{p}_{i}^{2}}{m_{i}}-(3 N+1) k_{B} T\right),
$$

where $Q$ is a parameter known as "thermal inertia" that throughout the work was set to $Q=0.05, k_{B}$ is the Boltzmann constant, $N$ is the number of particles and $T$ is the thermostat temperature.

For simplicity, we set $m_{i} \equiv m$ and consider reduced units, such that: mass is in units of $m$, distance in units of $\sigma$, and energy in units of $\epsilon$. The equations of motion are integrated using a fifth-order predictor-corrector algorithm, with a time step $d t=10^{-5}$ and we run the simulation up to $t=165$.

To generate the initial configurations, all particles were released within the region delimited by the dashed lines in Fig. 1. with an initial velocity drawn from a uniform distribution of zero mean. Particles are thermalized at the thermostat temperature within the dashed region, considering periodic boundary conditions along the horizontal direction and reflective top and bottom boundaries, without interacting with the channel walls. At $t=15$, the constraint imposed by the dashed lines is removed and particles move inside the channel, following the dynamics described by Eq. (4).

\section{RESULTS}

To characterize the effect of the asymmetry of the channel walls on the overall flux, we fixed $N=24, l=15$, $d=3$, and $h=6$, and performed simulations for different values of the asymmetry coefficient $\alpha \in[-1,1]$. To reduce statistical noise, instead of directly measuring the outlet flux $\phi(t)$, we introduce an integrated quantity $B(t)$, which we call balance. $B(t)$ is defined as the difference between the cumulative number of particles crossing the rightmost boundary from the left to the right and the ones crossing it in the opposite direction, up to time $t$. In the continuum limit,

$$
\phi(t)=\frac{1}{h} \dot{B}(t) .
$$

Asymptotically, we expect that the balance scales linearly in time, and so we estimated the flux from a linear regression fit of the curve $B(t)$ in the linear regime.

Figure 2(a) shows the balance as a function of time for different values of $\alpha$. Clearly, spontaneous flow emerges as a result of the asymmetry of the channel walls. For right symmetric channels $(\alpha>0)$, the flow is from the left to the right, while for left symmetric channels $(\alpha<0)$, the flow is in the opposite direction. Figure 2(a) also shows examples for $\alpha=\{0,0.8,1\}$, for a particle-wall cutoff distance of $5 \sigma$. These examples show that the observed rectification of the particle motion is still observed 

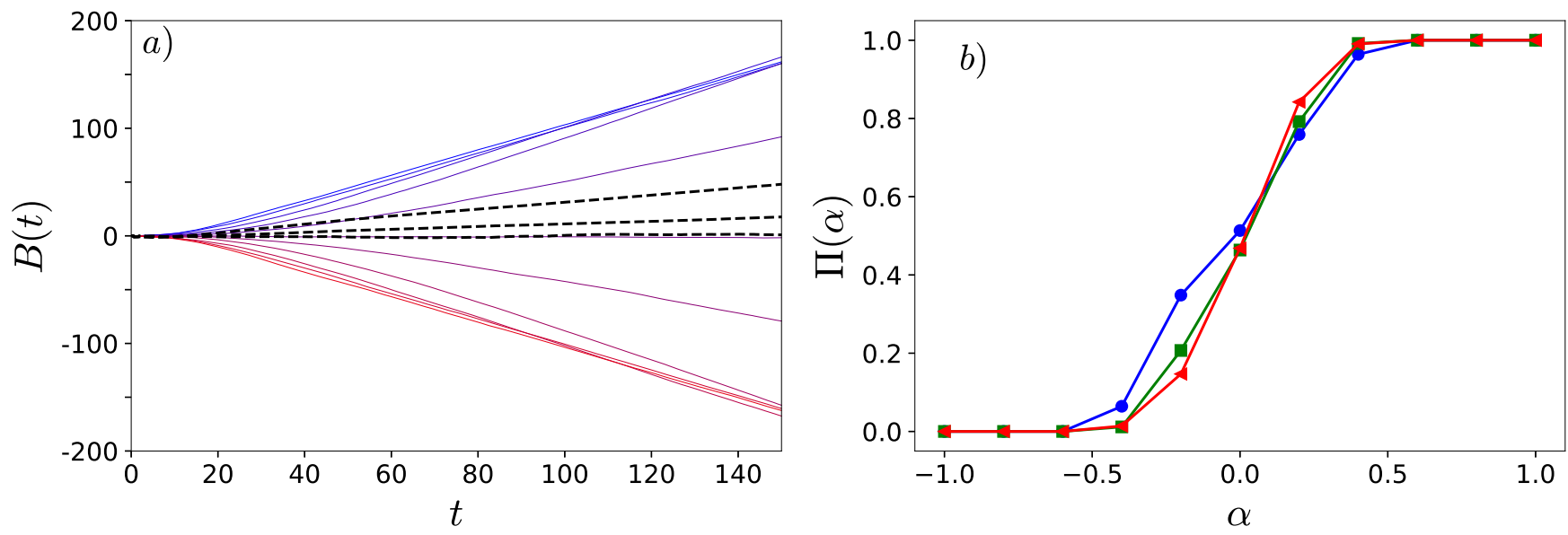

FIG. 2. (Color online) a) Time evolution of the balance $B(t)$ for different values of $\alpha=$ $\{-1.0,-0.8,-0.6,-0.4,-0.2,0,0.2,0.4,0.6,0.8,1.0\}$ (from bottom to top). Results are averages over 500 samples of systems of 100 particles at a thermostat temperature $T=2.5$ and $\gamma=1$. The black dashed lines correspond to simulations with $\alpha=\{0,0.8,1\}$ and $d_{c}=5 \sigma$ which show that the rectification phenomena is not unique to the choice $d_{c}=2.5 \sigma$ b) Fraction of samples $\Pi$ for which $B(t)>0$, for $T=2.5, \rho=0.143$, and $N=250$ (blue circles), $N=500$ (green squares), $N=1000$ (red triangles).
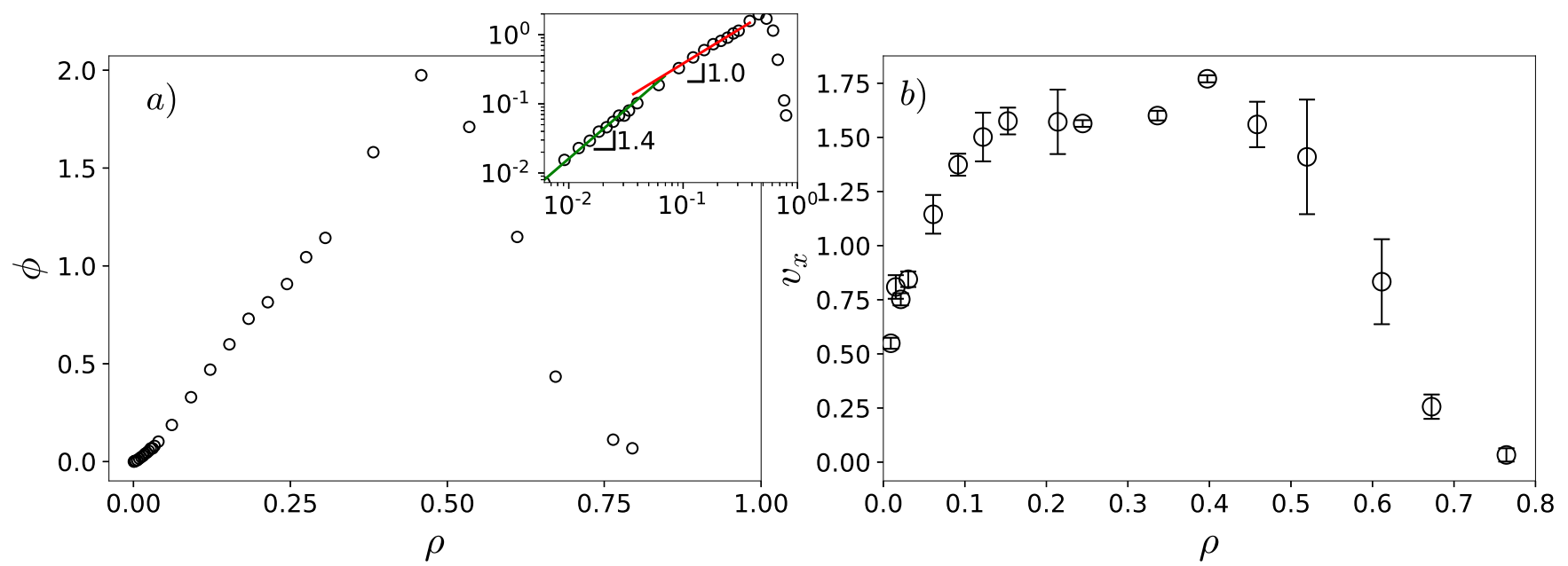

FIG. 3. (Color online) a) Flux $\phi$ as a function of the density $\rho$, for $T=2.5$ and $\alpha=1$. The two initial regimes are shown in the inset in a double logarithmic plot, where the dashed lines represent the power-law fits, $\phi \sim \rho^{\beta}$, with $\beta=1.47 \pm 0.07$ and $\beta=1.01 \pm 0.04$ for the low and intermediate density regimes, respectively. b) Average horizontal component of the particle velocity $\left(v_{x}\right)$ as a function of the density, for the same set of parameters.

for a larger cutoff distance, but the quantitative values of the flux are obviously different.

To analyze the transition at $\alpha=0$, we define $\Pi$ as the fraction of samples where $B(t)>0$ for large values of $t$ $(t=165)$. The dependence of $\Pi$ on $\alpha$ is in Fig. 2(b), for three different sizes of the channel. One sees that $\Pi$ is 0.5 for $\alpha=0$ and it seems to converge to a step function as the system size increases.

The dependence of the flux on the density is shown in Fig. 3(a), for $\alpha=1$ and $T=2.5$. One clearly observes an optimal density $\left(\rho_{\text {opt }} \approx 0.45\right)$ at which the flux is maximized. The data for $\rho<\rho_{\text {opt }}$ suggests two different regimes (see inset of Fig. 3(a)): a low-density regime, for $\rho<0.1$, and an intermediate-density regime, for $0.1<\rho<\rho_{\text {opt }}$. It is expected that the flux of particles is a monotonically increasing function of the density up to the point where it either saturates or start to decrease. For systems where the particle/particle collisions are more frequent than the particle/wall collisions, kinetic theory [35] suggests that the flux is linear in the density. However, for low densities, most of the particle collisions are with the wall; This implies that the flux is mostly determined by the chance of a particle to bounce off the wall and eventually cross the boundary at either 


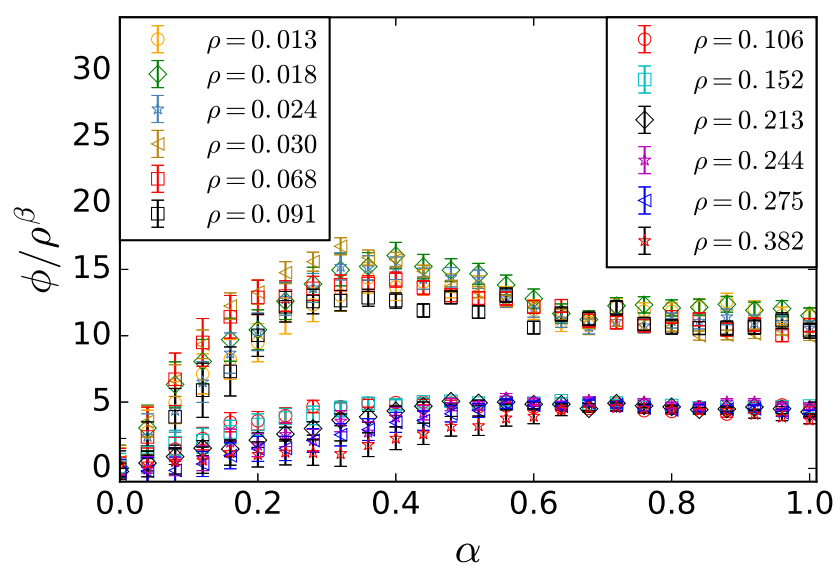

FIG. 4. (Color online) a) Flux $\phi$ rescaled by $\rho^{\beta}$ as a function of $\alpha$ for $T=2.5$ and different values of the density. The values reported on the left(right) legend were rescaled using $\beta=1.47(\beta=1.01)$.

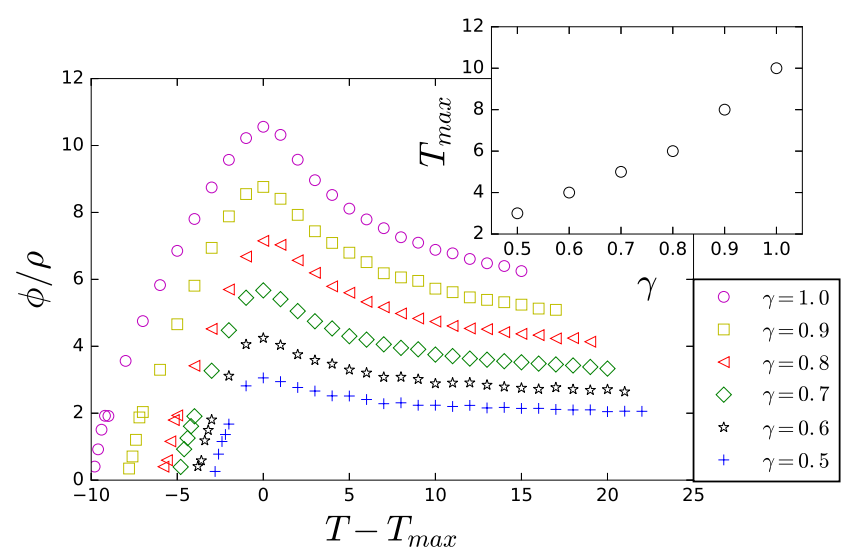

FIG. 5. (Color online) Scaled flux, $\phi / \rho$, as a function of the thermostat temperature, for $\alpha=1, \rho=0.0917$, and different values of the friction constant, $\gamma$. The thermostat temperature was shifted by $T_{\max }$, defined as the optimal temperature at which a maximum is observed in the flux. The dependence of $T_{\max }$ on $\gamma$ is shown in the inset.

end of the channel thus increasing or decreasing the net flux. In this case the monotonic increase of the flux is not necessarily linear. The numerical data suggest a power law scaling: $\phi \sim \rho^{\beta}$. Assuming a power-law scaling, we estimate $\beta=1.47 \pm 0.07$, for the low-density regime, and $\beta=1.01 \pm 0.04$, for the intermediate-density one.

Figure 3(b) shows the average horizontal component of the velocity $\left(v_{x}\right)$ as a function of the density. For the first regime, $v_{x}$ increases with the density thus, the enhancement of the flux with the density stems from an increase in the number particles per cell and possible collective effects affecting the particle velocity. Similar flux enhancement was reported in the context of comb systems, where the comb tooth would take the role of the sawtooth [36. However, in Ref. [36, the flux enhance- ment is observed for high densities and is related to the saturation of the traps. Here, instead, we observe a flux increase for much lower densities suggesting a different mechanism.

By contrast, for the intermediate-density regime, $v_{x}$ does not significantly change with the density and so the flux only increases due to an increase in the number of particles per cell, yielding a linear scaling. For $\rho>\rho_{\text {opt }}$, a third regime is observed, for which the flux simply decreases with the density due to crowding effects. That is, as the density increases, the available space for a particle to move diminishes. Thus, the motion of a single particle is strongly constrained by the presence of others. This in turn implies that for a particle to move over an extended region, it requires a concerted re-arrangement of several other particles. As the density increases, the chances that this concerted motion leads to a majority of particles moving in a preferred direction diminishes. Instead, it is more likely that the particles re-arrange by moving with no preferred direction leading to a decrease in the flux. Notice that this is consistent with the fact that $v_{x}$ approaches zero (Fig. 3(b)) which implies that roughly the same number of particles travel in each direction.

To study the dependence on $\alpha$, we plot in Fig. 4 , the flux rescaled by $\rho^{\beta}$, using the estimated values of $\beta$ for the corresponding regime. We observe a data collapse for each regime, suggesting that the power-law scaling is resilient over the entire range of values. The low density regime shows an optimal value of whereas the intermediate regime shows instead a nearly constant be- havior. We think that, for high-enough density, the rate of particle-particle collisions is significantly higher than the one of particle-wall collisions and thus the geometry of the walls does not play a significant role on the overall dynamics. By contrast, for low density, the rates of particleparticle and particle-wall collisions are comparable and a competition between the two is observed, leading to the maximum in the flux. Although there is some dispersion on the data collapse, it is clear that there are two distinct regimes. The origin of the dispersion can be due to finite-size effects or scaling corrections. The study of the nature of the transition and crossover between these two regimes requires further study, that is beyond the scope of this work. It is interesting to notice that the results for low densities in Fig. 4 are similar to those obtained by A. Sarracino 30. Namely, the quantity that indicates the presence of motion rectification ( $\phi$ in our case and $\langle V\rangle$ in Ref. [30]) shows a qualitatively similar non-monotonic behavior as a function of the asymmetry parameter. In both cases, it vanishes for the symmetric case and initially grows with the asymmetry, having a maximum for intermediate values, and then decreasing towards a saturation value for large values of the asymmetry parameter.

The dependence of the flux on the thermostat temperature is shown in Fig. 5, for different values of $\gamma$. For the entire range of values of $\gamma$, a maximum is observed at an optimal temperature, $T_{\max }$, that increases with $\gamma$ (see inset). Also, the optimal flux grows with dissipa- 
tion (increasing $\gamma$ ). Note that, for $\gamma=0$ the overall flux vanishes and thus a dissipative interaction with the walls is necessary to rectify the thermal motion of gas. This is consistent with the work of Prost et al. that suggests that time-reversal symmetry of trajectories needs to be broken to obtain rectification from asymmetric walls 37 .

Finally, to quantify the flux for a specific system, let us consider a channel of total length $L=216 \mu \mathrm{m}$, single cell length $l=9 \mu \mathrm{m}, \alpha=0, p=1.8 \mu \mathrm{m}$ and $h=3.7 \mu \mathrm{m}$ at room temperature, with colloidal particles of $\sigma=6 \times$ $10^{-7} \mathrm{~m}, m=2.49 \times 10^{-16} \mathrm{~kg}$, and $\gamma=2 \times 10^{-14} \mathrm{~kg} / \mathrm{s}[38$, 39. If we assume, $\epsilon / k_{b}=0.01414 \mathrm{~K}$ (where $k_{b}$ is the Boltzmann constant), we obtain a flow velocity $\phi / \rho \approx$ $42 \mu \mathrm{m} / \mathrm{s}$.

\section{CONCLUSION}

In this work, we systematically study the dependence of the rectification of the motion of a thermal gas on a channel of asymmetric dissipative walls. We found that the overall flux enhances with the friction constant of the particle/wall interaction and that it shows a nonmonotonic dependence on three other model parameters, namely, the thermostat temperature, channel asymme- try, and particle density. For the dependence of the flux on the density of particles, we found three different regimes. For low density, the flux scales superlinearly with the density, as collective effects lead also to an increase in the horizontal component of particle velocity. For intermediate density, the horizontal component of particle velocity saturates at a constant value and the overall flux scales linearly with the density. Finally, above an optimal value of the density, the flux monotonically decreases due to crowding effects. Future work might consider different geometries and a generalization to the three-dimensional case. The effect of different dissipation mechanisms as well as particle shapes are still open questions.

\section{ACKNOWLEDGMENTS}

We acknowledge financial support from the Brazilian institute INCT-SC and grant number FP7-319968 of the European Research Council. NA acknowledges financial support from the Portuguese Foundation for Science and Technology (FCT) under Contract no. UID/FIS/00618/2013 and from the Luso-American Development Foundation (FLAD), FLAD/NSF, Proj. $273 / 2016$.
[1] P. Hänggi and F. Marchesoni, Rev. Mod. Phys. 81, 387 (2009).

[2] P. Reimann, Phys. Rep. 361, 57 (2002).

[3] R. Feynman, R. B. Leighton, and M. L. Sands, The Feynman Lectures on Physics (Addison-Wesley, 1963) 3 volumes.

[4] H.Leff and A.Rex, Maxwell's Demon entropy, information and computing (Princeton University Press, 1990).

[5] P. Hänggi, F. Marchesoni, and F. Nori, Ann.Phys(Leipzig) 14, 51 (2005).

[6] I. Derényi and R. D. Astumian, Phys. Rev. E 58, 7781 (1998)

[7] C. Kettner, P. Reimann, P. Hänggi, and F. Müller, Phys. Rev. E 61, 312 (2000)

[8] S. Matthias and F. Müller, Nature 424, 53 (2003)

[9] J. Rousselet, L. Salome, A. Ajdari, and J. Prostt, Nature 370, 446 (1994)

[10] C. Marquet, A. Buguin, L. Talini, and P. Silberzan, Phys. Rev. Lett. 88, 168301 (2002)

[11] L. P. Faucheux, L. S. Bourdieu, P. D. Kaplan, and A. J. Libchaber, Phys. Rev. Lett. 74, 1504 (1995).

[12] J. Prost, J.-F. Chauwin, L. Peliti, and A. Ajdari, Phys. Rev. Lett. 72, 2652 (1994)

[13] G. Costantini, U. Marini Bettolo Marconi, and A. Puglisi, Phys. Rev. E 75, 061124 (2007)

[14] B. Cleuren and C. V. den Broeck, EPL 77, 50003 (2007)

[15] C. Van den Broeck, R. Kawai, and P. Meurs, Phys. Rev. Lett. 93, 090601 (2004)

[16] P. Eshuis, K. van der Weele, D. Lohse, and D. van der Meer, Phys. Rev. Lett. 104, 248001 (2010).
[17] A. Gnoli, A. Petri, F. Dalton, G. Pontuale, G. Gradenigo, A. Sarracino, and A. Puglisi, Phys. Rev. Lett. 110, 120601 (2013)

[18] R. Balzan, F. Dalton, V. Loreto, A. Petri, and G. Pontuale, Phys. Rev. E 83, 031310 (2011).

[19] S. Mobarakabadi, E. N. Oskoee, M. Schröter, and M. Habibi, Phys. Rev. E 88, 042201 (2013)

[20] L. Angelani, R. Di Leonardo, and G. Ruocco, Phys. Rev. Lett. 102, 048104 (2009)

[21] R. Di Leonardo, L. Angelani, D. DellArciprete, G. Ruocco, V. Iebba, S. Schippa, M. P. Conte, F. Mecarini, F. De Angelis, and E. Di Fabrizio, Proceedings of the National Academy of Sciences 107, 9541 (2010)

[22] A. Sokolov, M. M. Apodaca, B. A. Grzybowski, and I. S. Aranson, Proceedings of the National Academy of Sciences 107, 969 (2010)

[23] H. Li and H. P. Zhang, EPL 102, 50007 (2013).

[24] C. L. N. Oliveira, A. P. Vieira, D. Helbing, J. S. Andrade, and H. J. Herrmann, Phys. Rev. X 6, 011003 (2016).

[25] V. Holubec, A. Ryabov, M. H. Yaghoubi, M. Varga, A. Khodaee, M. E. Foulaadvand, and P. Chvosta, Entropy 19, 119 (2017).

[26] A. Ryabov, V. Holubec, M. H. Yaghoubi, M. Varga, M. E. Foulaadvand, and P. Chvosta, Journal of Statistical Mechanics: Theory and Experiment 2016, 093202 (2016).

[27] A. Sarracino, A. Gnoli, and A. Puglisi, Phys. Rev. E 87, 040101 (2013)

[28] G. Costantini, U. M. B. Marconi, and A. Puglisi, EPL (Europhysics Letters) 82, 50008 (2008)

[29] J. Talbot and P. Viot, Phys. Rev. E 85, 021310 (2012) 
[30] A. Sarracino, Phys. Rev. E 88, 052124 (2013).

[31] A. Baule and P. Sollich, EPL (Europhysics Letters) 97, 20001 (2012)

[32] M.P.Allen and D.J.Tildesley, Computer simulation of liquids (Clarendon press, 1989).

[33] T.Poschel and T. Schwager, Computational Granular Dynamics (Springer Berlin Heidelberg, 2005).

[34] W. G. Hoover, Phys. Rev. A 31, 1695 (1985).

[35] C. Cercignani, in The Boltzmann Equation and Its Applications (Springer, 1988) pp. 40-103.

[36] O. Bénichou, P. Illien, G. Oshanin, A. Sarracino, and R. Voituriez, Phys. Rev. Lett. 115, 220601 (2015)
[37] J. Prost, J. F. Chauwin, L. Peliti, and A. Ajdari, Phys. Rev. Lett. 72, 2652 (1994).

[38] A. Tomilov, A. Videcoq, M. Cerbelaud, M. A. Piechowiak, T. Chartier, T. Ala-Nissila, D. Bochicchio, and R. Ferrando, The Journal of Physical Chemistry B 117, 14509 (2013)

[39] A. Tomilov, A. Videcoq, T. Chartier, T. Ala-Nissil, and I. Vattulainen, The Journal of Chemical Physics 137, 014503 (2012) 